\title{
Temperature Dependence of Phonons on Stepped Surfaces Studied with Molecular Dynamics
}

\author{
Daniel D. Koleske and Steven J. Sibener \\ Department of Chemistry and The James Franck Institute, The University of Chicago, \\ Chicago, IL 60637
}

\begin{abstract}
With the aid of a new molecular dynamics (MD) program, we have calculated the phonon spectrum for the stepped Lennard-Jones (LJ) surfaces (533) and (511). We observe surface phonons on both systems. On the $L J(533)$ surface at $\bar{Q}=\overline{X A}$, we also observe modes localized at the step, identical in character to the step modes investigated by Knipp. ${ }^{8}$ These edge modes, $E_{1}$ and $E_{2}$, involve motions of atoms perpendicular to the step edge and are observed by resolving each of the four terrace atoms' contributions to the spectrum. The observation of step modes in MD simulations is also important, since only single force constant models in the harmonic approximation have previously been used to calculate phonons on slepped surfaces. ${ }^{5-8}$ We have also investigated the the temperature dependence of the z-polarized surface and step modes as a function of temperature on LJ(533). As the temperalure is raised, the step and surface modes decrease and broaden in frequency. For a surface temperature equal to $15 \%$ of the LJ bulk melting point $T_{m}$, the step modes are 4 times broader than in the low temperature simulations (about $3 \% \mathrm{Tm}$ ), while the surface modes are only 1.7 times broader. At these elevated temperatures, the step modes also couple strongly to the surface modes, creating a broad energy band on all four terrace atoms. This mode coupling may be important when considering vibrational energy flow on stepped surfaces, as well as the rate enhancements that catalytic reactions have on such surfaces as compared to their low Miller index counterparts.
\end{abstract}

Surface atoms located in terraces typically interact with $1 / 4$ to $7 / 12$ fewer atoms as compared to their bulk counterparts. This change in coordination dramatically modifies the electron density in the interfacial region, resulting in new chemical and physical properties at the surface. ${ }^{1}$ To date, most experimental and theoretical studies on the dynamical properties of surfaces have focussed on the characteristics of low Miller index surfaces (LMIS). ${ }^{1}$ Even greater departures form bulk-line behavior are expected to occur at sites of still lower coordination, such as at stepped surfaces [high Miller index surfaces (HMIS)]. In this paper we examine using molecular dynamics simulations, vibrational properties of two 
vicinal fcc surfaces. Temperature dependant runs will be shown to yield new information on how anharmonicity influences the vibrational dynamics of these model stepped surfaces.

Step atom bonding changes were observed in two previous EELS experiments. ${ }^{2,3}$ In one study on Pt(332), a mode at $205 \mathrm{~cm}^{-1}$ was observed at $\Gamma$, which is $15 \mathrm{~cm}^{-1}$ above the bulk band frequency for $\mathrm{Pt}^{2}$ This mode was modeled by stiffening the force constant between the step atom and the substrate. Ab initio calculations later confirmed this force constant stiffening, since the step atom was found to relax inward toward the step edge. 4 In contrast to this, a softened force constant was used to explained why a step mode on $\mathrm{TiC}(310)$ was observed below the surface phonon frequency. ${ }^{3}$ Other theoretical studies on stepped surfaces using single force constant models have explored the frequency and polarization of modes on a variety of stepped surfaces. ${ }^{5-7}$ Recently, using a Greens function method, Knipp has observed that some stepped surfaces have step localized modes which are confined to the step edge. ${ }^{8}$ Step localized modes provide localized vibrational states that adsorbates might couple with, assisting catalytic reactions, and opening up new energy flow pathways. ${ }^{9}$

We are currently investigating stepped surface vibrations using a newly developed molecular dynamics (MD) simulation program. Traditionally, surface phonons are calculated by either a slab calculation (SC) or a Greens function (GF) technique, rather than using computer intensive MD simulations. However, GF techniques become difficult as the size of the basis set increases and SC become time consuming when a large number of layers needed to converge the dynamical matrix. Also, both GF and SC require precise knowledge of the atomic positions, otherwise the force constant fit is arbitrary. However, the atoms relax to their equilibrium positions during the MD simulation guided by the model potential. Also, SC and GF methods require only small atomic displacements, unless anharmonic terms are added as a perturbation, whereas MD simulations automatically include the full anharmonic part of the potential. Since the anharmonic part is included, temperature dependent studies can be performed on the surface modes. We will present evidence which suggests that as the temperature is raised, the step modes, $E_{n}$, broaden in energy more that the surface modes, $S_{n}$. We will also show that at elevated temperatures these step modes 
couple strongly to the surface modes which are more localized in the middle of the terrace.

We will describe more details of both the program and further results on LMIS and HMIS in another paper. 10 The MD simulation program was designed to integrate Newton's equations of motion, $\mathbf{F}=$ ma. Greater than $95 \%$ of the computer run time is typically spent calculating these forces. A seventh order predictor-corrector method is used, 11 with the forces being calculated only once every time step. At the start of each simulation, the initial conditions of the parallelpiped slab are established, two sides of which are free surfaces, and the four remaining sides are surrounded by periodic boundary conditions. 12 Next, a table of nearest neighbors is established out to a particular radius around each atom. In this paper, the cutoff radius is set to include 140 neighbor interactions. Finally, a temperature is chosen for the system and the velocities are scaled accordingly. ${ }^{12}$ The integration of the coupled differential equations then proceeds until equilibrium is reached (about 1000-5000 time steps). ${ }^{10}$ When equilibrium is reached, the program calculates the $\bar{Q}$ resolved velocity vectors for 4096 time steps. After the program is completed, these vectors are then autocorrelated via a fast Fourier transform technique. With the integration time and time step size we used, an energy resolution of $0.1923 \omega$ is achieved, where $\omega=\sqrt{\varepsilon / M \sigma^{2}}$ ( $\varepsilon$ is the well depth, $M$ is the mass and $\sigma$ is the radius at which the potential is a minimum). ${ }^{11}$ The surface phonon spectra for both surfaces are then added together. In this paper, all MD spectra are shown as they appear after the transform, i. e. no Gaussian filtering is used.

We have not yet specified the type of potential used in the MD simulation. Currently, we are using a Lennard-Jones (LJ) 6-12 potential. We are not restricted to this potential, since changing the potential requires changing one subroutine. It is well understood that Lennard-Jones potentials do not model metals or semiconductors well. However, they have a simple analytical form and their surface phonon dispersion curves have been previously calculated.$^{13}$ We have calculated the LMIS using LJ potentials, ${ }^{10}$ and find that the MD simulations agree well with the SC. We hope to eventually model materials with more semi-empirical potentials, such as the Embedded Atom Method ${ }^{14}$ and Finnis Sinclair potentials ${ }^{15}$, which have predicted the structure and dynamics of clean metal surfaces. 
After these initial investigations to check the code, the low temperature phonon spectrum for the $L J(533)$ and the $L J(511)$ surface were calculated, using both $S C$ and $M D$. In Figure 1, the surface atomic arrangement is shown as viewed from the terrace normal or the $\mathbf{z}$ direction. On both surfaces, the $\mathbf{x}$ direction ( $y$ direction) was chosen to be perpendicular (parallel) to the step edge. Both surfaces have a c-rectangular surface Brillouin zone (SBZ), 5 which is also shown in Figure 1. The MD surface phonon spectra for both surfaces are shown in Figure 2, for $\bar{Q}=\overline{X A}$. For the (533), two different size simulations were used, one with a total of 4464 atoms (144 on each surface) and another with 8400 atoms (240 on each surface), to check if the periodic boundary conditions affect the phonon energy. In these two different sized systems, the polarizations of the modes agreed. However, the phonon energies of the 4464 atom system were 3-5\% lower in energy than the 8400 atom system. For the low temperature work only two runs were needed to resolve the phonon features.

FCC (533)

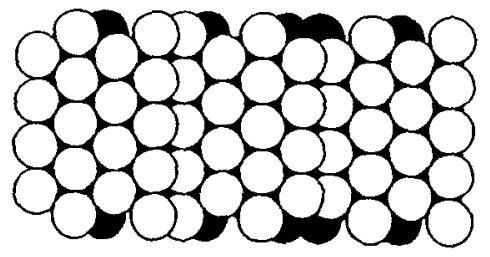

FCC (511)
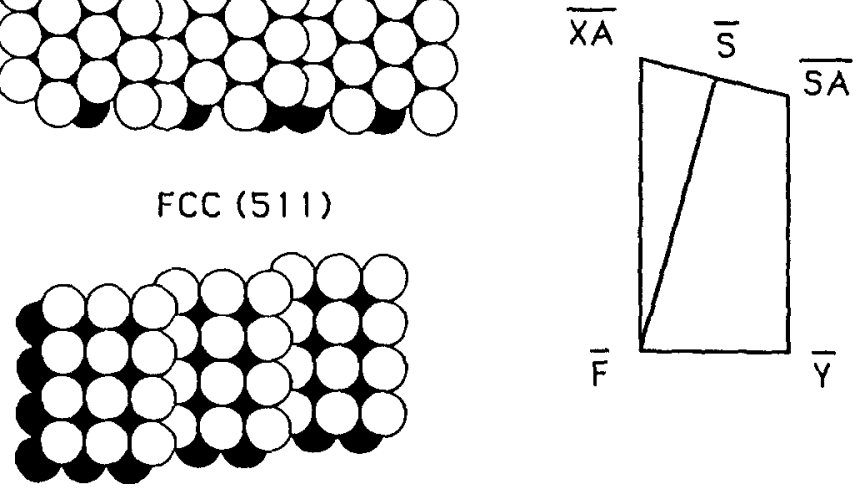

Figure 1: The surface geometries for the (533) and the (511) surfaces are shown, along with the Surface Brillouin Zone, which has a similar shape for both surfaces. Also, the coordinate directions are shown, with $z$ normal to the (111) or (100) plane, and $x(y)$ direction perpendicular (parallel) to the step edge. 
a) Lu(511) at XA TEP=0.62

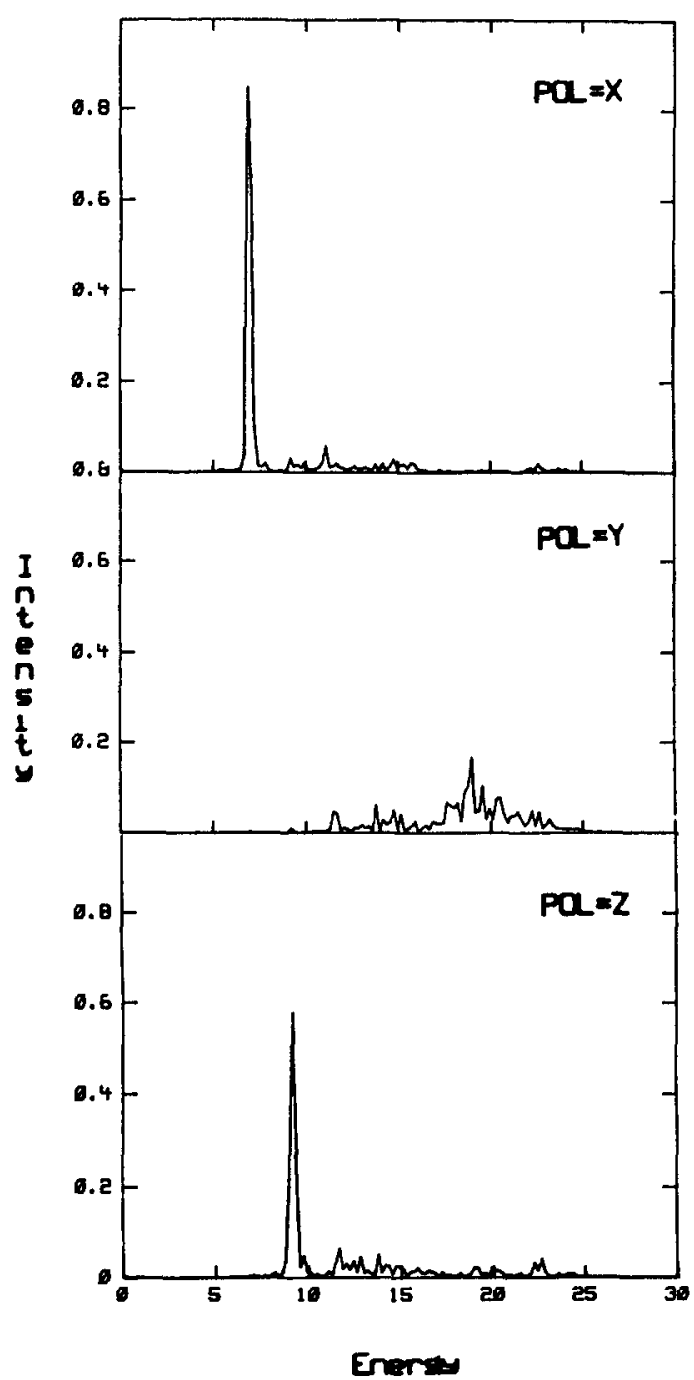

b) LJI533) at XA TEMP=0.62

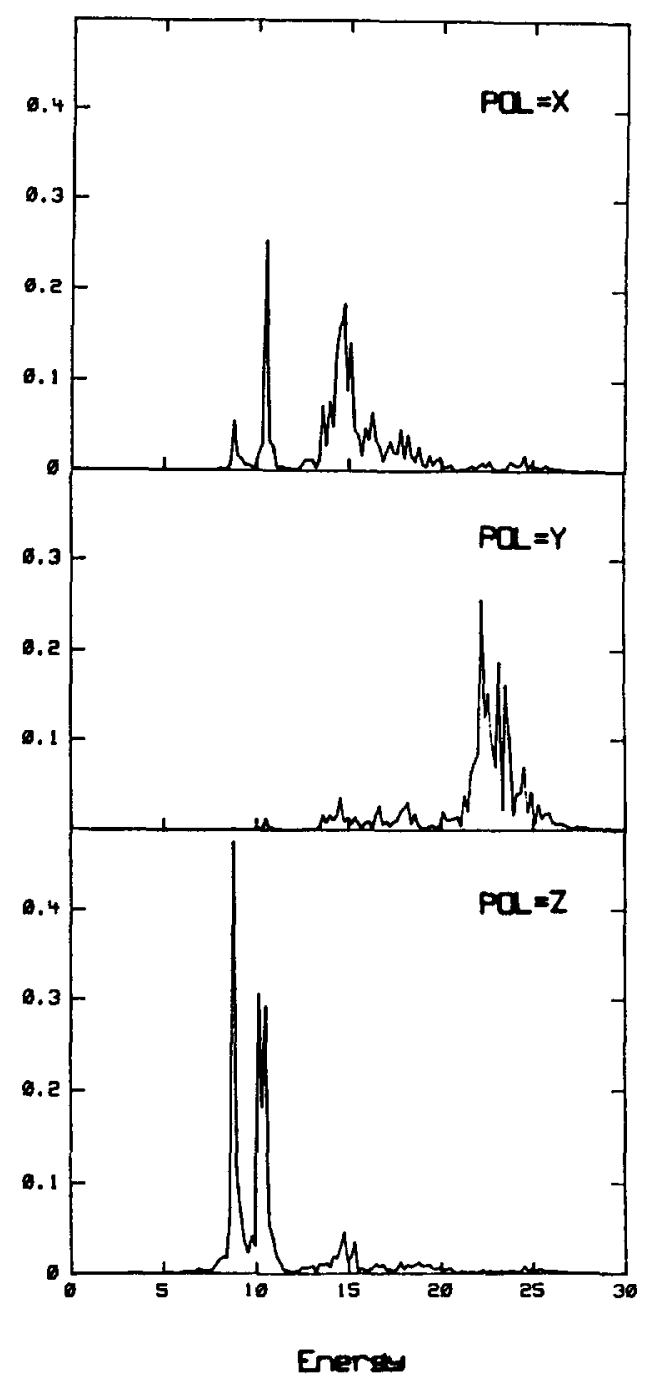

Figure 2: a) The (511) surface phonon spectrum at $\overline{X A}$ for the $x, y$ and $z$ polarlztions at a low simulation temperature. b) same as (a) except (533). 
The surface phonon spectra for the $L J(511)$ at $\overline{X A}$ are shown in Figure 2a for all three directions. Two modes are observed, one at $9.20 \mathrm{\omega}$ with $\mathrm{z}$-polarization and another at $6.90 \mathrm{\omega}$ with $\mathrm{x}$-polarization. These modes are similar to the $S_{4}$ and $S_{1}$ modes on $L J(100),{ }^{13}$ and they will not be discussed any further. No step modes are observed on LJ(511), in agreement with Knipp's results. ${ }^{8}$ The surface phonon spectra for $L J(533)$ at $\overline{X A}$ are plotted in Figure 3 for all three directions for the 8400 atom system. In these spectra it appears that three modes are observed that are z-polarized, a band of modes near 14-16 $\omega$ have $x$-polarization, and four modes between 22-24 $\omega$ with $y$-polarization. We will concentrate on the z-polarized modes, since they are well under the bulk band modes. Two of these z-polarized modes are close in energy to the Rayleigh wave, $S_{1}$, and the "folded" Rayleigh wave, $S_{1}$. One step mode, $E_{1}$, is found below the Rayleigh wave. However, near $\overline{X A}, S C$ predict a total of four modes: two Rayleigh modes and two edge modes, $E_{1}$ and $E_{2}$.

To further investigate this discrepancy, we have plotted in Figure $3 a$ the $z$-polarized spectra for the four terrace atoms. In the inset of each spectra, the atomic position for each layer is designated by a darkened circle. In Figure 3a, the first layer atom appears to have 3 modes, $E_{1}=8.84 \omega$ with a width of $(0.21 \omega)$, $E_{2}=10.53 \omega(0.18 \omega)$ and a bulk resonance at $14.41 \omega(0.89 \omega)$, which is stronger in the $x$-polarized spectra (not shown). Both step modes also are $x$-polarized, indicating that they vibrate perpendicular to the step edge. In the second layer the $S_{1}$, mode is observed at $10.33 \omega$ $(0.34 \omega)$, and in the third layer the $S_{1}$ mode is observed at $10.15 \omega$ $(0.34 \omega)$. These modes are about twice the energy resolution of $0.1928 \omega$, which is surprising, but with thought should be expected since the terraces are not very large. Hence the terrace vibrations cannot propagate very far before running into a step. In the fourth layer, two modes are observed, one which is $E_{1}$ at $8.84 \omega(0.25 \omega)$ and another at $14.41 \omega(0.89 \omega)$, whose origin was explained earlier. From Figure 3a, it is evident that four z-polarized modes are observed, with the largest spectral intensity for the $S_{1}$ mode being on the second layer, the largest spectral intensity for the $S_{1}$. mode on the third layer, and the largest spectral intensity for the $E_{1}$ and $E_{2}$ modes on the first and fourth layers. 
a) LJ (530) TEMP=0.02

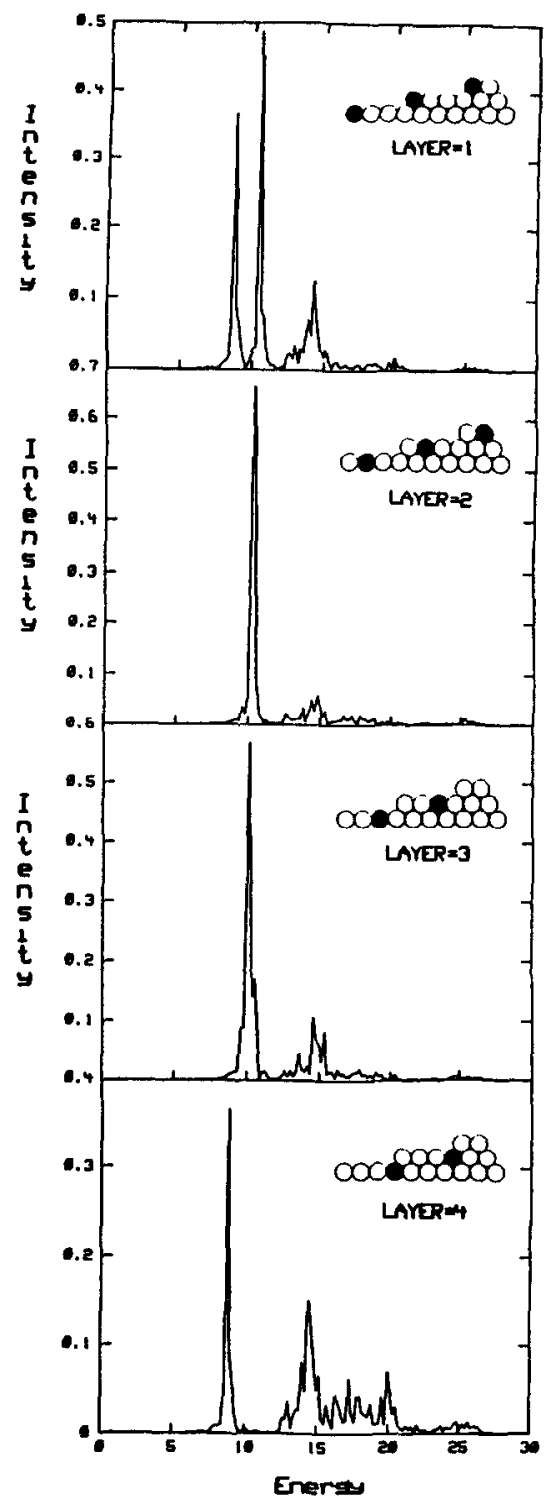

b) LJ(533) TEMP $=0.10$

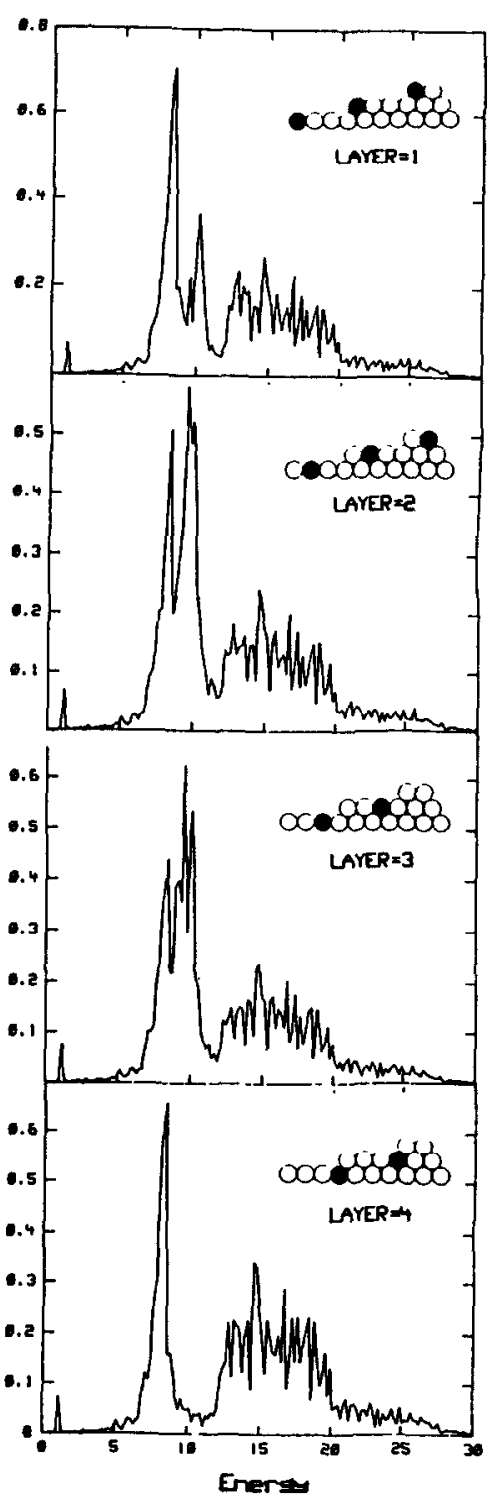

Figure 3: a) The atom resolved phonon spectrum at $\overline{X A}$ for modes with 2 polarization at a low simulation temperature. b) On the left side of this Figure the temperature is $15 \%$ of the melting point of solid Lennard-Jonesium. The diagram in the upper right hand corner denotes the position and layer of each terrace atom. 
Next, the lattice constant and the temperature of the simulation slab were increased in order to study how increased thermal motion influences these modes. In Figure $3 b$ we have plotted the zpolarized phonon spectra for each layer for a surface temperature equal to $15 \%$ of the bulk melting temperature, $\mathrm{T}_{\mathrm{m}} \cdot{ }^{12}$ These simulations used 4464 (144 surface) atoms. The spectra are a sum of six different runs. In the LJ solid, anharmonic effects are not observed until the solid is heated to $1 / 3 \mathrm{~T}_{\mathrm{m}}$, while anharmonic effects on surfaces are apparent at $1 / 6 \mathrm{~T}_{m} \cdot{ }^{12,16}$ However, at $T=15 \% \mathrm{~T}_{m}$ the step modes, $E_{n}$, are four times wider than in the low temperature simulations, while the surface modes, $S_{n}$, are only 1.7 times wider. This increase in the step mode width occurs because atoms at the step edge interact with fewer nearest neighbors. Also apparent in Figure 4 is that the $E_{n}$ modes are strongly coupled to the $S_{n}$, especially in layers two and three. This mode coupling is particularly interesting since it increases the number of low frequency modes.

In Figure 4 we have plotted the energy width of the $E_{1}, E_{2}, S_{1}$, and $S_{1}$ ' modes as a function of temperature, where the temperature

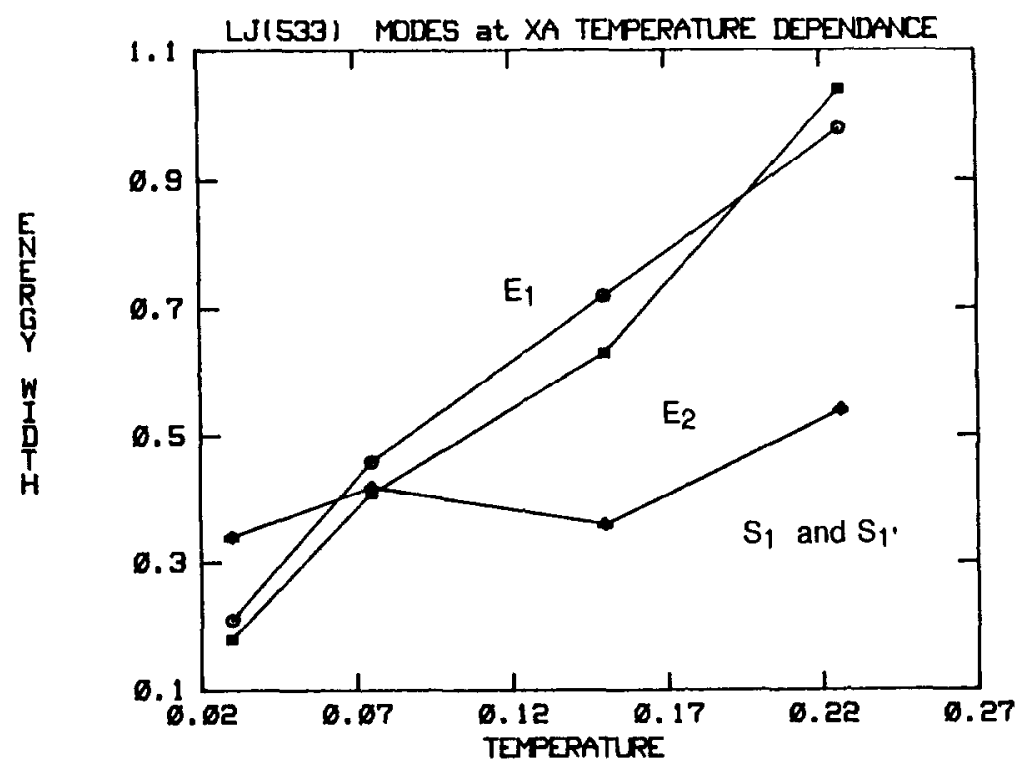

Figure 4: Plotted are the energy widths of the $E_{1}, E_{2}, S_{1}$, and $S_{1}$, as a function of temperature. For all four temperature, the width of $S_{1}$, and $S_{1}$ ' are identical, so only three lines are observed. 
$E_{1}$ and $E_{2}$ modes can be seen, while the widths of the $S_{1}$, and $S_{1}$. modes increase only slightly. All four modes also decrease in frequency, and this decrease is about the same amount for each temperature.

In conclusion, we have reported MD simulations on two slepped surfaces, $L J(533)$ and $L J(511)$, where we have extracted the surface vibrational modes at $\mathbf{Q}=X A$. We have shown on the $L J(533)$ surface at this $\overline{\mathbf{Q}}$, that two surface and two step modes exist, and have shown on which atoms they reside. As the temperature of the surface is increased, the edge modes broaden more than the surface modes. We have also shown that as the temperature is further increased, the edge modes, $E_{n}$, couple strongly to the surface modes, $S_{n}$, creating a wide energy band. This last observation may be crucial when considering energy flow pathways for stepped surfaces, and the enhanced catalytic activity of stepped surfaces as opposed to their LMI counterparts.

\section{Acknowledgements}

We acknowledge many helpful conversations with $P$. A. Knipp on his work, which included a preprint of his thesis. We also thank D. Padowitz, L. Brown and S. King. This work was supported, in part, by the Air Force Office of Scientific Research grant and the National Science Foundation Materials Research Laboratory at the University of Chicago.

\section{References}

1. For a recent review, see J. E. Black, Structure and Dynamics of Surfaces I, edited by W. Schommers and P. von Blankenhagen (Springer-Verlag, Berlin, 1986), p. 153; or see, Phonons 89 , edited by S. Hunklinger, W. Ludwig, and G. Weiss, (World Scientific, Singapore, 1990), p. 841.

2. H. Ibach and D. Bruchmann, Phys. Rev. Lett. 41, 958 (1978).

3. M. Wuttig, C. Oshima, T. Aizawa, R. Souda, S. Otami, and Y. Ishizawa, Surf. Sci. 193,180 (1988).

4. Mark Mostoller and Uzi Landmann, Phys. Rev. B 20, 1755 (1979).

5. J. E. Black, and P. Bopp, Surf. Sci. 140, 275 (1984).

6. G. Armand, and P. Masri, Surf. Sci. 130, 89 (1983).

7. A. Lock, J. P. Toennies, and G. Witte, Phonons 89, edited by S. Hunklinger, W. Ludwig, and G. Weiss, (World Scientific, Singapore, 1990), p. 901.

8. P. Knipp, Phys. Rev. B 40, 7993 (1989); preprint of thesis paper submitted to Phy. Rev. B.

9. E. W. Montrol, and R. B. Polts, Phys. Rev. 102, 72 (1956).

10. D. D. Koleske, and S. J. Sibener, to be published. 
11. W. E. Milne, Numerical Solutions of Differential Equations, (Dover, New York, 1970).

12. W. Schommers, Structure and Dynamics of Surfaces I, edited by W. Schommers and $P$. von Blankenhagen (Springer-Verlag, Berlin, 1986), p. 199.

13. R. E. Allen, G. P. Alldredge, and F. W. de Wette, Phys. Rev. B. 4, 1648; Phys. Rev B. 4, 1661; Phys. Rev B. 4, 1682 (1971).

14. J. S. Nelson, E. C. Sowa, and M. S. Daw, Phys. Rev. B. 40, 1465 (1989).

15. M. W. Finnis and J. E. Sinclair, Phil. Mag. A. 50, 45 (1984).

16. A. R. McGurn, A. A. Maradudin, R. F. Wallis, and A. J. C. Ladd, Phys. Rev. B 37, 3964 (1988). 